\title{
On the Optimal Insulation of Conductors ${ }^{1}$
}

\author{
S. J. Cox, ${ }^{2}$ B. KAwOHL, ${ }^{3}$ AND P. X. UhLIG ${ }^{4}$ \\ Communicated by K. A. Lurie
}

\begin{abstract}
We coat a conductor with an insulator and equate the effectiveness of this procedure with the rate at which the body dissipates heat when immersed in an ice bath. In the limit, as the thickness and conductivity of the insulator approach zero, the dissipation rate approaches the first eigenvalue of a Robin problem with a coefficient determined by the shape of the insulator. Fixing the mean of the shape function, we search for the shape with the least associated Robin eigenvalue. We offer exact solutions for balls; for general domains, we establish existence and necessary conditions and report on the results of a numerical method.
\end{abstract}

Key Words. Two-phase conductors, eigenvalues, reinforcements, boundary conditions of the third type.

\section{Introduction}

We suppose that a homogeneous conductor occupies the smooth openbounded connected set $\Omega \in \mathbb{P}^{d}$. With $\epsilon>0$ and a nonnegative function $h$ on $\partial \Omega$, whose outer normal we denote by $n$, we define the insulated conductor

$$
\Omega_{\epsilon}(h) \equiv \bar{\Omega} \cup\{x+\delta h(x) n(x): x \in \partial \Omega, 0<\delta<\epsilon\} .
$$

If the conductivity of $\Omega$ is 1 , while that of $\Omega_{\varepsilon}(h) \backslash \Omega$ is $\epsilon$, then in solving the heat equation on $\Omega_{\epsilon}(h)$, subject to zero Dirichlet data, one is led to consider

\footnotetext{
'This work was conducted while the first author was visiting the Mathematisches Institut, Universität Köln with the generous support of the Alexander von Humboldt Foundation.

${ }^{2}$ Associate Professor, Department of Computational and Applied Mathematics, Rice University, Houston, Texas.

${ }^{3}$ Professor, Mathematisches Institut, Universität Köln, Köln, Germany.

${ }^{4}$ Assistant Professor, Mathematics Department, St. Mary's University, San Antonio, Texas.
} 
the eigenvalue problem

$$
\begin{aligned}
& -\nabla \cdot\left(1_{\Omega}+\left(1-1_{\Omega}\right) \epsilon\right) \nabla u=\lambda u, \quad x \in \Omega_{\epsilon}(h), \\
& u=0, \quad x=\partial \Omega_{\epsilon}(h),
\end{aligned}
$$

where $1_{\Omega}$ denotes the characteristic function of $\Omega$. In particular, the least eigenvalue, denoted by $\lambda_{1}(\Omega, h, \epsilon)$, governs the rate at which $\Omega_{\epsilon}(h)$ dissipates heat and, hence, is a natural measure of the quality of the insulation procedure. Thus, we seek out those $h$ that render $\lambda_{1}(\Omega, h, \epsilon)$ small. Our search is considerably simplified if we first permit the thickness and conductivity of the insulator to simultaneously approach zero. More precisely, we recall that Friedman (Ref. 1) has established that

$$
\lambda_{1}(\Omega, h, \epsilon) \rightarrow \xi_{1}(\Omega, h), \quad \text { as } \epsilon \rightarrow 0,
$$

where $\xi_{1}(\Omega, h)$ is the least eigenvalue of the Robin problem,

$$
\begin{aligned}
& -\Delta u=\xi u, \quad x \in \Omega, \\
& u+h \partial u / \partial n=0, \quad x \in \partial \Omega .
\end{aligned}
$$

Friedman went on to pose the problem of minimizing $\xi_{1}(\Omega, h)$ over those $h$ of fixed mean. The present paper provides the solution to this problem under the additional asumption that $h$ satisfies a pointwise lower bound. More precisely, we address

$$
\hat{\xi}_{1}(\Omega)=\inf _{h \in \mathrm{ad}_{0}(\partial \Omega)} \xi_{1}(\Omega, h),
$$

where

$$
\operatorname{ad}_{0}(\partial \Omega) \equiv\left\{h: \alpha|\Omega| /|\partial \Omega| \leq h(x), \int_{\partial \Omega} h d x=h_{0}|\Omega|\right\}
$$

and $0<\alpha<h_{0}<\infty$ are both fixed throughout. As $h$ has the dimension of length, the convention specified in (3) renders $\alpha$ and $h_{0}$ dimensionless.

Let us dispense with a few preliminaries before summarizing the main results. To begin, the weak formulation of (1) reads

$$
\int_{\Omega} \nabla u \cdot \nabla v d x+\int_{\partial \Omega} h^{-1} u v d x=\xi \int_{\Omega} u v d x, \quad \forall v \in H^{1}(\Omega),
$$

and gives rise to the associated variational characterization,

$$
\begin{aligned}
& \xi_{1}(\Omega, h)=\inf _{u \in H_{l}(\Omega)} \mathscr{R}(u, h), \\
& \mathscr{R}(u, h) \equiv \int_{\Omega}|\nabla u|^{2} d x+\int_{\partial \Omega} h^{-1} u^{2} d x,
\end{aligned}
$$


where $H_{1}^{1}(\Omega)$ is the class of $H^{1}(\Omega)$ functions with $L^{2}(\Omega)$ norm one. It follows from (5) [see, e.g., Gilbarg and Trudinger (Ref. 2, Theorem 8.38)] that $\xi_{1}(\Omega, h)$ is simple and may be associated with a nonnegative eigenfunction. A useful lower bound on $\xi_{1}(\Omega, h)$ follows from the Hölder inequality

$$
\int_{\partial \Omega} h^{-1} u^{2} d x \geq\left(\int_{\partial \Omega} u d x\right)^{2} / \int_{\partial \Omega} h d x .
$$

For, using (6) in (5), one finds

$$
\begin{array}{r}
\xi_{1}(\Omega, h) \geq \inf _{u \in H_{i}^{\prime}(\Omega)} \int_{\Omega}|\nabla u|^{2} d x+\left(1 / h_{0}|\Omega|\right)\left(\int_{\partial \Omega} u d x\right)^{2}, \\
\forall h \in \operatorname{ad}_{0}(\partial \Omega) .
\end{array}
$$

If we denote by $\zeta_{1}\left(\Omega, h_{0}\right)$ the value of this infimum and by $v_{1}$ a function at which this infimum is attained, we find $\zeta_{1}\left(\Omega, h_{0}\right)$ and $v_{1}$ to be the first eigenpair of the nonlocal problem

$$
\begin{aligned}
& -\Delta v=\zeta v, \quad \text { in } \Omega, \\
& h_{0}|\Omega| \partial v / \partial n+\int_{\partial \Omega} v d x=0, \quad \text { on } \partial \Omega
\end{aligned}
$$

In Section 2, we find that

$$
\zeta_{1}\left(B, h_{0}|B| /|\partial B|\right)=\xi_{1}\left(B, h_{0}|B| /|\partial B|\right),
$$

when $B$ is a ball, and so establish that an insulator of constant thickness is optimal for such domains. In Section 3, we show via the direct method that the infimum in (2) is attained for arbitrary smooth $\Omega$. We produce the associated necessary conditions in Section 4; in Section 5, we describe and test a numerical method.

Finally, let us recall that Buttazzo (Ref. 3) has investigated the related problem of choosing $h$ so to maximize the heat $\int_{\Omega} w f d x$, where $w$ is the temperature of the insulated body in the presence of the heat source $f$; i.e., $w$ satisfies

$$
\begin{aligned}
& -\Delta w=f, \quad x \in \Omega, \\
& w+h \partial w / \partial n=0, \quad x \in \partial \Omega .
\end{aligned}
$$

Our analyses differ in that Buttazzo was able to establish existence of an optimal $h$ without imposing a pointwise lower bound; i.e., he permits $\alpha=$ 0 . He produced explicit solutions for rods subject to nonnegative $f$ and for planar annular domains under constant $f$. He did not address the numerical 
treatment of more general domains. It is exactly out of numerical necessity that we have imposed a pointwise lower bound on $h$. More precisely, the lack of a lower bound on $h$ introduces infinite components [see, e.g., (17)] in the stiffness matrix stemming from the standard finite-element approximation to (4). Moreover, our numerical results suggest that the bound $\alpha$ may always be chosen small enough, with respect to $h_{0}$, to guarantee that the best $h$, in the sense of (2), remains positively above $\alpha$.

\section{Best Insulation of Balls}

If $\Omega=B_{a}$ is a ball of radius $a$ in $\mathbb{R}^{d}$, then each radial solution of (8) is necessarily of the form

$$
v(r ; \zeta)=(\sqrt{\zeta} r)^{1-d / 2} J_{d / 2-1}(\sqrt{\zeta} r)
$$

where $\zeta$ is a zero of

$$
z \mapsto v(a ; z)+h_{0}\left(\left|B_{a}\right| /\left|\partial B_{a}\right|\right) d v / d r(a ; z) .
$$

Let us denote these zeros, in increasing order, by $\left\{\zeta_{0, j}\right\}_{j=1}^{\infty}$. Note that these are exactly the radial eigenfunctions and associated eigenvalues of (1) with

$$
h=h_{0}\left|B_{a}\right| /\left|\partial B_{a}\right| \text {. }
$$

Next, we note that every nonradial Neumann eigenfunction of the Laplacian on $B_{a}$ is also an eigenfunction of (8). If $v$ is an eigenfunction of (8) that is not a nonradial Neumann eigenfunction, then perforce it is orthogonal to each of these and, hence, lies in the span of the radial Neumann eigenfunctions, the upshot being that $v$ is radial. Having already characterized the radial eigenfunctions, it follows that the spectrum of (8) is

$$
\left\{\zeta_{0, j}: j=1, \ldots, \infty\right\} \cup\left\{\mu_{i, j}: i=1, \ldots, \infty, j=1, \ldots, \infty\right\},
$$

where the $\mu_{i, j}$ are Neumann eigenvalues (nondecreasing in $j$ for fixed $i$ ) associated with nonradial eigenfunctions. It is not difficult to show that $\zeta_{0,1}$ is the smallest member of the spectrum; therefore, $\zeta_{1}=\zeta_{0,1}$. Observing now that (8) and (1) with $h=h_{0}\left|B_{a}\right| /\left|\partial B_{a}\right|$ have precisely the same radial eigenfunctions and associated eigenvalues, we find

$$
\xi_{1}\left(B_{a}, h_{0}\left|B_{a}\right| /\left|\partial B_{a}\right|\right)=\zeta_{1}\left(B_{a}, h_{0}\left|B_{a}\right| /\left|\partial B_{a}\right|\right) .
$$

This fact, together with (7), yields the following proposition. 
Proposition 2.1. Insulators of constant thickness are best for balls. More precisely,

$$
\xi_{1}\left(h_{0}\left|B_{a}\right| /\left|\partial B_{a}\right|, B_{a}\right) \leq \xi_{1}\left(h, B_{a}\right), \quad \text { for all } h \in \operatorname{ad}_{0}\left(\partial B_{a}\right) .
$$

\section{Existence of Optimal Insulators}

The arguments of the previous section were, of course, custom fit to balls. Although

$$
\zeta_{1}\left(\Omega, h_{0}\right) \leq \xi_{1}(\Omega, h), \quad \text { for all } h \in \operatorname{ad}_{0}(\partial \Omega),
$$

indeed holds for all smooth domains $\Omega$, our ignorance of $\zeta_{1}\left(\Omega, h_{0}\right)$, together with our doubt that equality may be attained for domains other than the disk, leads us to pursue less constructive means in the proof of existence of minimal $h$. In the following, we shall drop the argument $\Omega$, when the domain is understood. For example, $\hat{\xi}_{1}$ will denote the value of the infimum in (2).

Proposition 3.1. If $\Omega$ is smooth, open, bounded, and connected, then there exists an $\hat{h} \in \operatorname{ad}_{0}(\partial \Omega)$ such that $\xi_{1}(\hat{h})=\hat{\xi}_{1}$.

Proof. Suppose that $\left\{h_{n}\right\} \subset \operatorname{ad}_{0}(\partial \Omega)$ is a minimizing sequence, i.e., $\xi_{1}\left(h_{n}\right) \rightarrow \hat{\xi}_{1}$. As $\left\{h_{n}^{-1}\right\}$ is uniformly bounded in $L^{\infty}(\partial \Omega)$, it has a subsequence, that we neglect to relabel, that converges in the weak ${ }^{*}$ topology to some function $g$, i.e., $h_{n}^{-1} * g$. We write $h \equiv g^{-1}$ and suppose that $u_{n}$ is the positive first eigenfunction, associated with $h_{n}$, normalized such that

$$
\int_{\Omega} u_{n}^{2} d x=1, \quad \int_{\Omega}\left|\nabla u_{n}\right|^{2} d x+\int_{\partial \Omega} h_{n}^{-1} u_{n}^{2} d x=\xi_{1}\left(h_{n}\right) .
$$

From (9), it follows that $\left\{u_{n}\right\}_{n}$ is bounded in $H^{1}(\Omega)$ and, hence, that $u_{n} \rightarrow u$ in $H^{1}(\Omega), u_{n} \rightarrow u$ in $L^{2}(\Omega)$, and the traces $\left.\left.u_{n}\right|_{\partial \Omega} \rightarrow u\right|_{\partial \Omega}$ in $L^{2}(\partial \Omega)$. These observations permit us to pass to the limit in the weak form

$$
\int_{\Omega} \nabla u_{n} \cdot \nabla v d x+\int_{\partial \Omega} h_{n}^{-1} u_{n} v d x=\xi_{1}\left(h_{n}\right) \int_{\Omega} u_{n} v d x,
$$

and so conclude that $\hat{\xi}_{1}$ and $u$ constitute an eigenpair for $h$. As $u$ is positive, it follows that $\hat{\xi}_{1}=\xi_{1}(h)$. It remains only to check whether $h$ lies in $\mathrm{ad}_{0}$. The pointwise bound offers no obstacle. Regarding the integral constraint, we note that the convexity of $t \mapsto t^{-1}$ allows us to invoke Dacorogna (Ref. 4, 
Theorem 1.1) in claiming that

$$
\int_{\partial \Omega} h d x=\int_{\partial \Omega} g^{-1} d x \leq \liminf _{n} \int_{\partial \Omega}\left(h_{n}^{-1}\right)^{-1} d x=h_{0}|\Omega| .
$$

Hence, at worst, $h$ uses less than all the available insulator. Now, let $\hat{h}$ be any function that is pointwise greater than $h$ but that lies in $\operatorname{ad}_{0}$. From (5), it follows that $\xi_{1}(\hat{h}) \leq \xi_{1}(h)$. Now, as $\hat{\xi}_{1}=\xi_{1}(h)$, our claim follows.

\section{Necessary Conditions}

We denote by $\hat{u}_{1} \in H_{1}^{1}(\Omega)$ the positive eigenfunction associated with $\hat{h}$; note that one may always interchange the order of two minimizations, and so record

$$
\xi_{1}(\hat{h})=\mathscr{R}\left(\hat{u}_{1}, \hat{h}\right)=\min _{h \in \mathrm{d}_{0}} \min _{u \in H_{l}^{!}(\Omega)} \mathscr{R}(u, h)=\min _{u \in H_{1}^{\dagger}(\Omega)} \min _{h \in \mathrm{ad}_{0}} \mathscr{R}(u, h)
$$

In other words,

$$
\mathscr{R}\left(\hat{u}_{1}, \hat{h}\right)=\min _{u \in H_{1}^{1}(\Omega)} \mathscr{R}(u, \hat{h}), \quad \mathscr{R}\left(\hat{u}_{1}, \hat{h}\right)=\min _{h \in \mathrm{ad}_{0}} \mathscr{R}\left(\hat{u}_{1}, h\right) .
$$

The former simply states that $\hat{u}_{1}$ is an eigenfunction corresponding to $\hat{h}$. The latter, however, informs us that $\hat{h}$ solves the much simpler optimization problem

$$
\int_{\partial \boldsymbol{\Omega}} \hat{h}^{-1} \hat{u}_{1}^{2} d x=\min _{h \in \mathrm{ad}_{0}} \int_{\partial \Omega} h^{-1} \hat{u}_{1}^{2} d x .
$$

We make it still simpler by exchanging the volume constraint for a Lagrange multiplier. More precisely, from the Lagrange multiplier rule of Clarke (Ref. 5, Theorem 6.1.1), we deduce that (10) implies the existence of $v_{1} \geq 0$ and $\left|v_{1}\right|+\left|v_{2}\right|>0$ such that

$$
\int_{\partial \Omega} \hat{h}^{-1}\left(v_{1} \hat{u}_{1}^{2}+v_{2} \hat{h}^{2}\right) d x=\min _{h \in \mathrm{ad}} \int_{\partial \Omega} h^{-1}\left(v_{1} \hat{u}_{1}^{2}+v_{2} h^{2}\right) d x,
$$

where

$$
\operatorname{ad} \equiv\{h: \alpha|\Omega| /|\partial \Omega| \leq h(x)\} .
$$

From $v_{1} \hat{u}_{1}^{2} \geq 0$, we deduce from (11) that $v_{2}<0$. More precisely, if $v_{2}>0$, then the term in parentheses in (11) is positive, and so (11) requires that $\hat{h} \equiv \infty$, in contradiction of the integral constraint. 
Similarly, as $v_{2}<0$ if $v_{1}=0$, then (11) implies that

$$
\hat{h} \equiv \alpha|\Omega| /|\partial \Omega|,
$$

again in violation of the integral constraint.

As a result, $v^{2} \equiv-v_{2} / v_{1}$ is well defined and we may deduce from (11), along the lines say of Cea and Malanowski (Ref. 6), the following pointwise necessary conditions:

$$
\begin{aligned}
& \alpha|\Omega| /|\partial \Omega|=\hat{h}(x) \Rightarrow \hat{u}_{1}(x) \leq v \hat{h}(x), \\
& \alpha|\Omega| /|\partial \Omega| \leq \hat{h}(x) \Rightarrow \hat{u}_{1}(x)=v \hat{h}(x) .
\end{aligned}
$$

With respect to the best insulated ball, where $\hat{h}$ and the trace of $\hat{u}_{1}$ are constant, we note that (13) is trivially satisfied along the entire boundary. In the general case, these conditions state that, where $\hat{h}$ is off of its bound, it coincides with (a multiple of) its associated eigenfunction $\hat{u}_{1}$. Recalling (1), this implies that

$$
\partial \hat{u}_{1} / \partial n=\text { const }
$$

there. Conditions on $\Omega$ sufficient to guarantee the solvability (together with questions pertaining to the inheritance of symmetry) of $-\Delta u=\xi u$ subject to (14) on $\partial \Omega$ have yet to be specified.

\section{Numerical Approach}

As the analytical necessary conditions fail to characterize the optimal $h$, we turn to its numerical approximation. Our approach is similar to that promoted by Cox and Uhlig (Ref. 7).

We write $\partial \Omega$ as the closure of the disjoint union of $m$ open edges $\left\{\Gamma_{j}\right\}_{j=1}^{m}$ and restrict $h$ to

$$
h(s)=\sum_{j=1}^{m} H_{j} 1_{\Gamma_{j}}(s)
$$

where $H \in \mathbb{R}^{m}$ satisfies

$$
\alpha|\Omega| /|\partial \Omega| \leq H_{j}, \quad \sum_{j=1}^{m} H_{j}\left|\Gamma_{j}\right|=h_{0}|\Omega| .
$$

In order to compute $\xi_{1}$ at such an $h$, we restrict our search to eigenvectors of the form

$$
u(x)=\sum_{i=1}^{p} U_{i} T_{i}(x)
$$


where $p<\infty$ and each $T_{i}$ is a piecewise linear hat function. On substituting this expansion into the weak form (3), with $v$ running through the $T_{i}$, we arrive at the $p \times p$ eigensystem

$$
(K+Q(H)) U=\Xi M U,
$$

where $K$ and $M$ are independent of $H$ while

$$
Q_{i j}(H)=\int_{\partial \Omega} T_{i} T_{j} / h d x=\sum_{k=1}^{m}\left(1 / H_{k}\right) \int_{\Gamma_{k}} T_{i} T_{j} d s
$$

Let us denote the least eigenvalue of $(16)$ by $\Xi_{1}(H)$. As this approximation procedure respects the symmetry of the original problem, we retain a variational characterization,

$$
\begin{aligned}
& \Xi_{1}(H)=\min _{U \cdot M U=1} \mathscr{R}(U, H), \\
& \mathscr{R}(U, H) \equiv U \cdot K U+U \cdot Q(H) U .
\end{aligned}
$$

As the approximation is refined (i.e., as $m$ and $p$ become large), $\Xi_{1}(H)$ tends to $\xi_{1}(h)$ and so $\Xi_{1}(H)$ may be assumed simple. Recalling Cox (Ref. 8), this permits the computation of the gradient of $\Xi_{1}$ in terms of the representation in (18). More precisely,

$$
\partial \Xi_{1}(H) / \partial H_{k}=U_{H} \cdot\left[\partial Q(H) / \partial H_{k}\right] U_{H},
$$

where $U_{H}$ is the minimizer (eigenvector) in (18) associated with $\Xi_{1}(H)$, and recalling (17),

$$
\partial Q_{i j}(H) / \partial H_{k}=-\left(1 / H_{k}^{2}\right) \int_{\Gamma_{k}} T_{i} T_{j} d s .
$$

With $\mathrm{AD}_{0}$ denoting those $H$ satisfying (15), we have now assembled all the ingredients necessary for a numerical attack on the finite-dimensional optimization problem

$$
\min _{H \in \mathrm{AD}_{0}} \Xi_{1}(H) \text {. }
$$

We have implemented such an attack in Matlab, using the routine pdeeig, within its Partial Differential Equations Toolbox, in the computation of $\Xi_{1}(H)$ and $U_{H}$, and the routine constr, within its Optimization Toolbox, in the resolution of (19). We present detailed results for two model domains.

In Fig. 1, we have plotted a rounded equilateral triangle together with its associated best insulation for the parameter choice

$$
\alpha=0.1, \quad h_{0}=0.25 \text {. }
$$




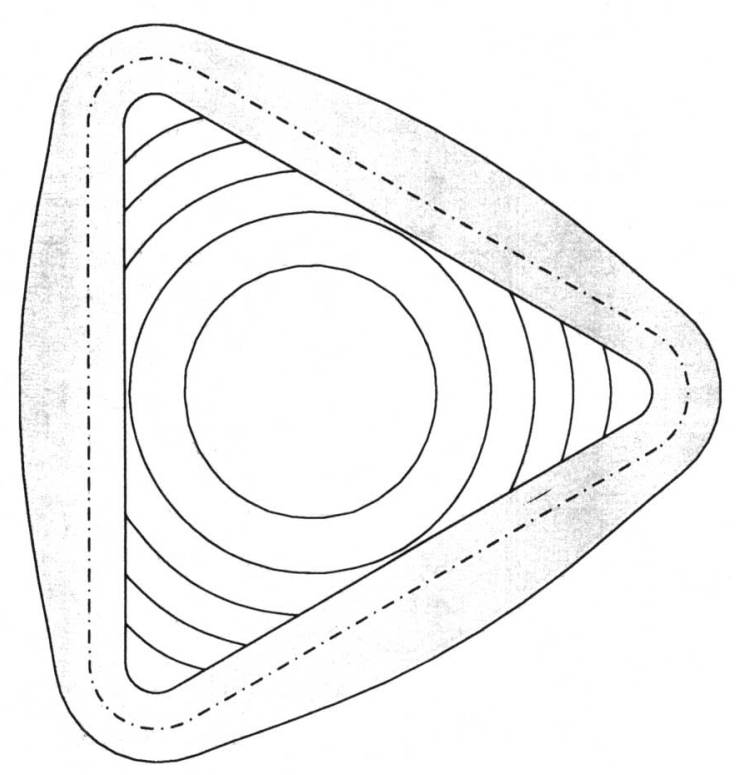

Fig. 1. Best insulation, $\alpha=0.1$.

The best $H$ is depicted here by the shaded region. We have magnified it in order to better depict its variation in thickness. The contours within the domain are those of the associated eigenvector $U_{H}$. The dot-dash line within the insulated region corresponds to the line

$$
h(x)=\alpha|\Omega| /|\partial \Omega| .
$$

Hence, given (20), the best $H$ achievable from a random start lies completely off of its lower bound. We have found that, by increasing $\alpha$, one can arrive at an $H$ that attains $\alpha$. In particular, with

$$
\alpha=0.2, \quad h_{0}=0.25,
$$

the best insulation of the rounded equilateral triangle assumes the form depicted in Fig. 2. For each of these runs, we partitioned the boundary into $m=60$ segments and partitioned the domain into $p=1177$ elements.

As our final example, we depart from convexity and consider the rounded cross. We plot it in Fig. 3 along with its (magnified) best $H$ and associated $U_{H}$ contours under the parameter choice

$$
\alpha=0.05, \quad h_{0}=0.1 \text {. }
$$




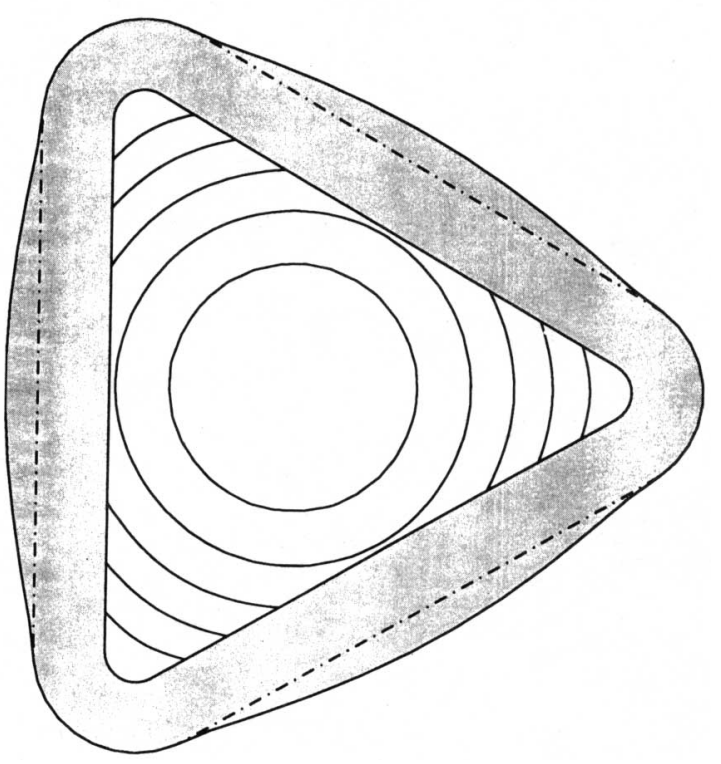

Fig. 2. Best insulation, $\alpha=0.2$.

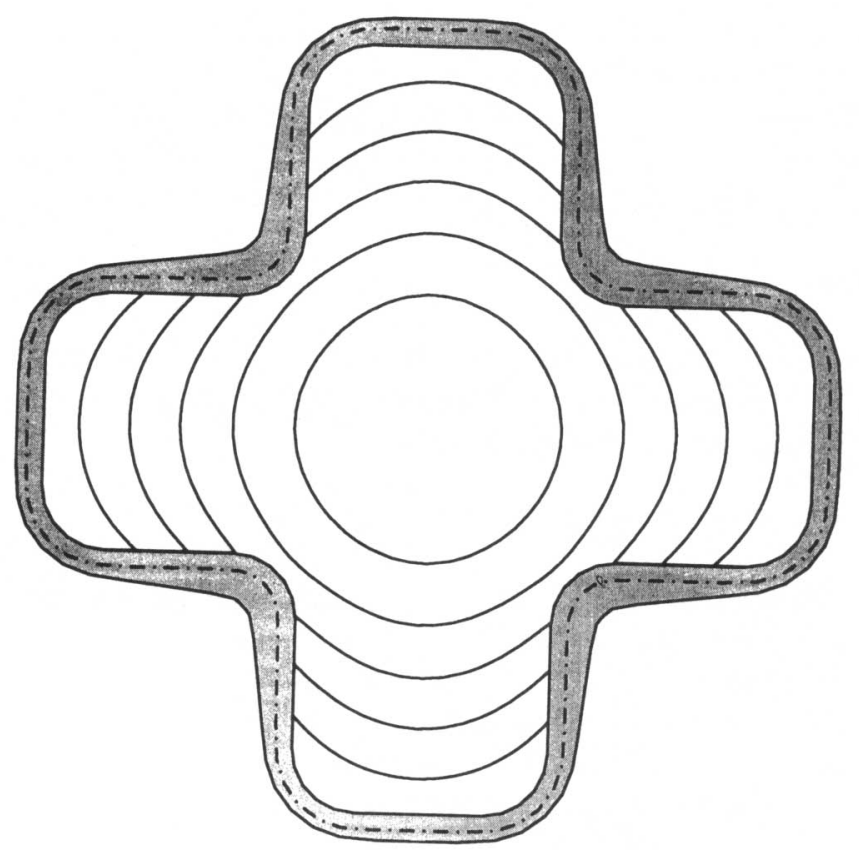

Fig. 3. Best insulation of a cross. 
As in the first case, the best insulator is everywhere off of its lower bound. In this case, we partitioned the boundary into $m=96$ segments and the domain into $p=1913$ elements.

\section{References}

1. Friedman, A., Reinforcement of the Principal Eigenvalue of an Elliptic Operator, Archive for Rational Mechanics and Analysis, Vol. 73, pp. 1-17, 1980.

2. Gilbarg, D., and Trudinger, N., Elliptic Partial Differential Equations of Second Order, 2nd Edition, Springer, Berlin, Germany, 1983.

3. Buttazzo, G., Thin Insulating Layers: The Optimization Point of View, Material Instabilities in Continuum Mechanics and Related Problems, Edited by J. M. Ball, Oxford University Press, Oxford, England, pp. 11-19, 1988.

4. Dacorogna, B., Weak Continuity and Weak Lower Semicontinuity of Nonlinear Functionals, Lecture Notes in Mathematics, Springer, Berlin, Germany, Vol. 922, 1982.

5. Clarke, F., Optimization and Nonsmooth Analysis, SIAM, Philadelphia, Pennsylvania, 1990.

6. Cea, J., and Malanowski, K., An Example of a Max-Min Problem in Partial Differential Equations, SIAM Journal on Control and Optimization, Vol. 8, pp. 305-316, 1970.

7. Cox, S. J., and UhLIG, P. X., Where Best to Hold a Drum Fast, SIAM Journal on Optimization, 1999.

8. Cox, S. J., The Generalized Gradient at a Multiple Eigenvalue, Journal of Functional Analysis, Vol. 33, pp. 30-40, 1995. 